EPJ manuscript No.

(will be inserted by the editor)

\title{
Beyond the quark model of hadrons from lattice QCD
}

\author{
Chris Michael \\ Theoretical Physics Division, Mathematical Sciences Department, University of Liverpool, Liverpool L69 3BX, UK. \\ Received: date / Revised version: date
}

\begin{abstract}
Lattice QCD can give direct information on OZI-violating contributions to mesons. Here we explore the contributions that split flavour singlet and non-singlet meson masses. I discuss in detail the spectrum and decays for scalar mesons (ie including glueball effects). I also review the status of hybrid mesons and their decays.
\end{abstract}

PACS. 12.38.Gc Lattice QCD calculations - 12.39.Mk Glueball and non-standard multi-quark/gluon states

\section{Introduction}

Lattice QCD is a first-principles approach to solving QCD non-perturbatively. It enables theorists to explore the consequences of QCD for quarks of different mass and so complements experimental studies. It is especially valuable for validitating phenomenological models. Direct comparison with experiment is hampered by two obstructions: (i) the continuum limit (lattice spacing $a \rightarrow 0$ ) should be taken but this is not computationally feasible in many cases and (ii) the $u$ and $d$ quark masses are too light to be successfully explored on a lattice so that one has to rely on chiral perturbation theory and extrapolate from heavier quark masses. Nevertheless, lattice QCD enables exploration of the hadron spectrum and matrix elements and can be used to quantify the success (and failure) of quark models. One area where the quark model is deficient is in flavour singlet mesons. Here the gluonic degrees of freedom are likely to make a significant contribution.

\section{Flavour singlet mesons}

In lattice QCD, one studies mesons by creating a quark anti-quark pair at time 0 with the quantum numbers of that meson and then annihilating the quark anti-quark pair at time $t$. The quark sources and sinks are then combined using the fully non-perturbative quark propagators determined on the lattice. For flavour singlet mesons, there will be two contributions: disconnected $D(t)$ and connected $C(t)$ as illustrated in fig. 1.

At large $t$ where ground state contributions dominate these measured correlations satisfy $C(t)=c e^{-m_{1} t}$ and $C(t)+D(t)=d e^{-m_{0} t}$ where $m_{0}$ is the flavour singlet mass and $m_{1}$ the flavour non-singlet mass. Then by a study of $D / C$ which is given by $(d / c) \exp \left(\left(m_{1}-m_{0}\right) t\right)-1$ one can explore the mass splitting between flavour singlet and

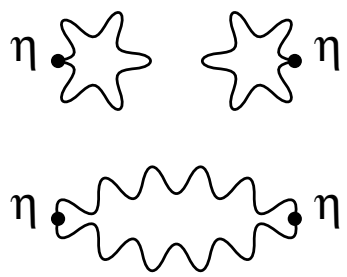

Fig. 1. The disconnected (above) and connected (below) correlators for flavour singlet mesons at time separation $t$. Here the wiggly lines represent full quark propagators. The illustration is for a pseudoscalar meson $(\eta)$ but the same diagrams apply to all mesonic quantum numbers.

non-singlet which is a measure of the OZI violating gluonic contribution arising from the disconnected diagram.

From a lattice study of this for all mesonic quantum numbers [i] the only significant disconnected contributions were found for scalar and for pseudoscalar mesons. This is not unexpected: both from the phenomenology of the observed meson masses and from the expectation that the lightest glueball has scalar quantum numbers and that topological charge fluctuations have pseudoscalar quantum numbers.

For a review of the situation concerning pseudoscalar mesons see ref [2]. We now discuss the glueballs and their impact on the meson spectrum.

\section{Glueballs and scalar mesons}

Glueballs are defined to be hadronic states made primarily from gluons. The full non-perturbative gluonic interaction is included in quenched QCD. In the quenched approximation, there is no mixing between such glueballs and quark - antiquark mesons. A study of the glueball spectrum in quenched QCD is thus of great theoretical value. 
Chris Michael: Beyond the quark model of hadrons from lattice QCD

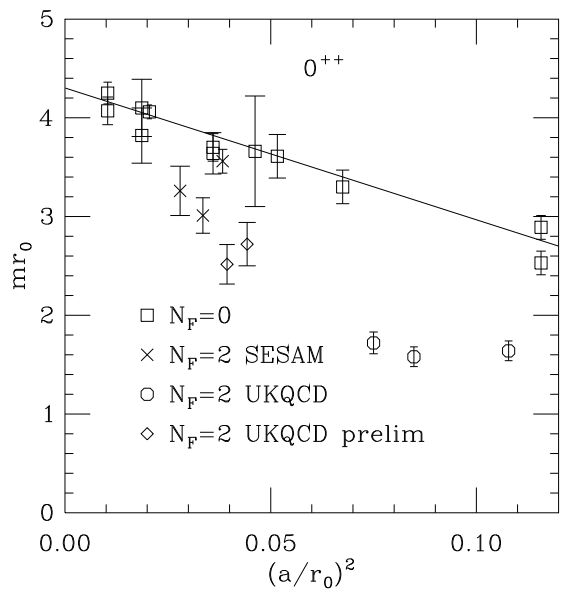

Fig. 2. The value of mass of the lightest $J^{P C}=0^{++}$meson

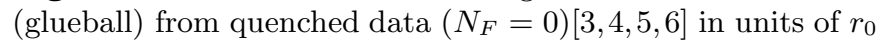
where $r_{0} \approx 0.5 \mathrm{fm}$. The straight line shows a fit describing the approach to the continuum limit as $a \rightarrow 0$. Results [0, 8, 10] for the lightest scalar meson with $N_{F}=2$ flavours of sea quarks are also shown.

This has been studied extensively [5, 11] and the consensus is that the lightest glueball has scalar quantum numbers with the tensor $\left(J^{P C}=2^{++}\right)$and pseudoscalar glueballs $\left(J^{P C}=0^{-+}\right)$next in mass. The quenched results have been explored to very small lattice spacings and there is convincing evidence that the continuum limit values have been extracted - see fig. 1 . Since the quenched approximation does not reproduce experiment, different ways to set the scale will differ - by $\pm 10 \%$. Using a conventional scale assignment $\left(r_{0}=0.5 \mathrm{fm}\right)$ gives masses of around $1.6 \mathrm{GeV}$ for the scalar glueball and $2.2 \mathrm{GeV}$ for the tensor.

One signal of great interest would be a glueball with $J^{P C}$ not allowed for $q \bar{q}$ - a spin-exotic glueball or oddball - since it would not mix with $q \bar{q}$ states. These states are found [4, 5, 11] to be high lying: considerably above $2 m\left(0^{++}\right)$. Thus they are likely to be in a region very difficult to access unambiguously by experiment.

Within the quenched approximation, the glueball states are unmixed with $q \bar{q}, q \bar{q} q \bar{q}$, etc. Furthermore, the $q \bar{q}$ states have degenerate flavour singlet and non-singlet states in the quenched approximation. This gives rise to anomalies in the quenched approximation: for example the scalar meson propagation can have the wrong sign 12 because the $\eta \pi$ intermediate state is mistreated. Once quark loops are allowed in the vacuum then these anomalies are removed. Indeed, for the favour-singlet states of any given $J^{P C}$, there will be mixing between the $s \bar{s}$ state, the $u \bar{u}+d \bar{d}$ state and the glueball. Since the scalar glueball is lightest, we expect the largest effects here and one can explore this by measuring directly the scalar mass eigenstates in a study with $N_{f}=2$ flavours of sea-quark.

Most studies have shown no significant change of the glueball spectrum as dynamical quarks are included [7]. However the larger lattice spacing result [8] shows a significant reduction in the lightest scalar mass, as shown in fig. 1. Before concluding that this implies a lower scalar mass in the continuum limit, one needs to check whether an enhanced order $a^{2}$ correction might be present. Studies using the same approach at a finer lattice spacing [9, 10] do suggest that this large order $a^{2}$ effect is significant, but studies even nearer to the continuum or with improved actions are needed to resolve this fully.

Let us now discuss the mixing of the scalar glueball and scalar mesons. In quenched QCD there is the problem described above concerning scalar mesons. For heavy enough quarks this is unimportant and one can measure the mixing strength on a quenched lattice even though no mixing actually occurs. On a rather coarse lattice $\left(a^{-1} \approx 1.2\right.$ $\mathrm{GeV}$ ), two groups have attempted this [13, 8]. Their results expressed as the mixing for two degenerate quarks of mass around the strange quark mass are similar, namely $E \approx 0.36 \mathrm{GeV}$ [13] and $0.44 \mathrm{GeV}$ [8]. Opinions differ [13, 8 as to whether this large mixing which would shift the glueball mass down by $20 \%$ will persist to the continuum limit.

From dynamical fermion studies with $N_{f}=2$, one can determine the flavour singlet and non-singlet mass spectrum. No glueball, as such, can be defined. What we find [8, 10] is that the lightest flavour-singlet scalar meson is lighter than the lightest flavour non-singlet. This is in qualitative agreement with the mixing scenario described above.

As well as this mixing of the glueball with $q \bar{q}$ states, there will be mixing with $q \bar{q} q \bar{q}$ states which will be responsible for the hadronic decays. A first attempt to study this [14] in quenched QCD yields an estimated width for decay to two pseudoscalar mesons from the scalar glueball of order $100 \mathrm{MeV}$. A more realistic study would involve taking account of mixing using gluonic, $q \bar{q}$ and $q \bar{q} q \bar{q}$ operators in full QCD.

\section{Hybrid mesons}

A hybrid meson has the gluonic degrees of freedom which are excited non-trivially. The most significant consequence of this, experimentally, will be mesons with $J^{P C}$ values not allowed in the quark model for a $q \bar{q}$ system (such as $\left.1^{-+}\right)$

On the lattice one can easily deal with relatively light quarks (down to the strange quark mass) or extremely heavy quarks (treated as static or using NRQCD). I first discuss hybrid mesons with static heavy quarks where the description can be thought of as an excited colour string. The lattice results [15, 16, 17] can then be presented as potential energy versus quark-antiquark separation $R$. The ground state will correspond to the usual interquark potential whereas excited states will have non-trivial representations of the symmetries, e.g. the lightest excited state $\left(\Pi_{u}\right)$ has colour flux which is a difference of paths from quark to antiquark of the form $\sqcap-\sqcup$.

From the potential corresponding to these excited gluonic states, one can determine the spectrum of hybrid quarkonia using the Schrödinger equation in the BornOppenheimer approximation. The $\Pi_{u}$ symmetry state will 
produce a degenerate set of eight hybrid mesons of which those with $J^{P C}=1^{-+}, 0^{+-}$and $2^{+-}$are spin-exotic and hence will not mix with $Q \bar{Q}$ states. They thus form a very attractive goal for experimental searches for hybrid mesons.

Within the quenched approximation, the lattice evidence for $b \bar{b}$ quarks points to a lightest hybrid spin exotic meson $\mathrm{H}$ with $J^{P C}=1^{-+}$at an energy given by [24] $\left(m_{H}-m_{2 S}\right) r_{0}=1.9 \pm 0.1$. This has been checked with $N_{f}=2$ studies 18,25] which find similar mass ratios $\left(m_{H}-m_{1 S}\right) /\left(m_{1 P}-m_{1 S}\right)$ but a larger splitting in terms of $r_{0}$, namely [18] $\left(m_{H}-m_{2 S}\right) r_{0}=2.4 \pm 0.2$. Using the experimental mass of the $\Upsilon(2 S)$, these results imply that the lightest spin exotic hybrid is in the mass range 10.7 to $10.9 \mathrm{GeV}$. Above this energy there will be many more hybrid states, many of which will be spin exotic.

Within this static quark framework, one can explore the decay mechanisms. One special feature is that the symmetries of the quark and colour fields about the static quarks must be preserved exactly in decay. This has the consequence that the decay from a $\Pi_{u}$ hybrid state to the open- $b$ mesons $\left(B \bar{B}, B^{*} \bar{B}, B \bar{B}^{*}, B^{*} \bar{B}^{*}\right)$ will be forbidden 25] if the light quarks in the $B$ and $B^{*}$ mesons are in an $\mathrm{S}$-wave relative to the heavy quark. The decay to $B^{* *}$-mesons with light quarks in a $\mathrm{P}$-wave is allowed by symmetry but not energetically. The only allowed decays are to $\chi_{b}+M$ where $M$ is a light quark-antiquark meson in a flavour singlet. Lattice estimates [25] of these transitions have been made and the dominant mode (with a width of around $100 \mathrm{MeV}$ ) is found to be with $M$ as a scalar meson. This decay analysis does not take into account heavy quark motion or spin-flip and these effects will be significantly more important for charm quarks than for b-quarks.

Several lattice groups [19, 20,21,22] have studied hybrid spectra for light quarks and find the lightest spinexotic hybrid to have $J^{P C}=1^{-+}$and mass (for $s$-quarks) of around $2 \mathrm{GeV}$. The corresponding light-quark $(u, d)$ state would be around $120 \mathrm{MeV}$ lighter. The light quark results have also been 20] extrapolated to charm quarks and masses near $4.4 \mathrm{GeV}$ are found for the corresponding state. A recent study has confirmed this value but with a very long extrapolation [22]. These mass estimates can be compared to naive estimates [16] of the spin-exotic charm state mass of $4.0 \mathrm{GeV}$ from the static quark approach which will have an uncontrolled systematic error.

It is not easy to reconcile these lattice results with experimental indications 23] for resonances at $1.4 \mathrm{GeV}$ and $1.6 \mathrm{GeV}$, especially the lower mass value. Mixing with $q \bar{q} q \bar{q}$ states such as $\eta \pi$ is not included for realistic quark masses in the lattice calculations. This can be interpreted, dependent on one's viewpoint, as either that the lattice calculations are incomplete or as an indication that the experimental states may have an important meson-meson component in them.

An attractive prospect to study hybrid mesons is from $p \bar{p}$ annihilation. Spin-exotic hybrids, which provide the best signal, will only be produced in association with other hadrons. The decay channel to $\chi_{c}+\pi+\pi$ with the two $\pi$ mesons in an S-wave is a promising detection channel. Lattice studies give first estimates of the masses and decay widths but more work needs to be done to constrain the systematic errors on these estimates.

\section{References}

1. UKQCD Collaboration, C. McNeile, C. Michael and K.J. Sharkey, Phys. Rev. D65, (2002) 014508.

2. UKQCD Collaboration; C McNeile and C Michael, Phys. Lett. B491, (2000) 123.

3. P. De Forcrand et al., Phys. Lett. B152, (1985) 107.

4. C. Michael and M. Teper, Nucl. Phys. B314 (1989) 347.

5. UKQCD collaboration, G. Bali et al., Phys. Lett. B309 (1993) 378.

6. H. Chen et al., Nucl. Phys. B (Proc. Suppl.) 34 (1994) 357; A. Vaccarino and D. Weingarten, Phys. Rev. D60 (1999) 114501.

7. SESAM and $\mathrm{T} \chi \mathrm{L}$ Collaboration, G. Bali et al., Nucl. Phys. B (Proc. Suppl.) 63 (1998) 209; Phys. Rev. D62 (2000) 054503

8. C. McNeile and C. Michael, Phys. Rev. D63, (2001) 114503

9. UKQCD Collaboration, A. Hart and M. Teper, Phys. Rev. D 65, (2002) 034502.

10. UKQCD collaboration; A. Hart, C. McNeile and C. Michael, (in preparation).

11. C. Morningstar and M. Peardon, Phys. Rev. D56 (1997) 4043; ibid., D60 (1999) 034509.

12. W. Bardeen et al., Phys. Rev. D65 (2002) 014509.

13. W. Lee and D. Weingarten, Nucl. Phys. B (Proc. Suppl) 63, 194 (1998); hep-lat/9805029; Phys. Rev. D61 (2000) 014015.

14. J. Sexton, A. Vaccarino and D. Weingarten, Nucl. Phys. B (Proc. Suppl.) 42 (1995) 279; Phys. Rev. Lett. 75 (1995) 4563

15. L.A. Griffiths, C. Michael and P.E.L. Rakow, Phys. Lett. B129 (1983) 351.

16. S. Perantonis and C. Michael, Nucl. Phys. B347 (1990) 854.

17. K. Juge, J. Kuti and C. Morningstar, Phys. Rev. Lett. 82 (4400) 1999; Nucl. Phys. B (Proc. Suppl) 83 (2000) 304.

18. CP-PACS Collaboration, T. Manke et al., Phys. Rev. Lett. 82 (1999) 4396; Phys. Rev. D64 (2001) 097505;

19. UKQCD Collaboration, P. Lacock, C. Michael, P. Boyle and P. Rowland, Phys. Rev. D54 (1996) 6997; Phys. Lett. B401 (1997) 308.

20. C. Bernard et al., Phys. Rev. D56 (1997) 7039; Nucl. Phys. B (Proc. Suppl.) 73 (1999) 264.

21. P. Lacock and K. Schilling, Nucl. Phys. B (Proc. Suppl.) 73 (1999) 261.

22. Z.H. Mei and X.Q. Luo, hep-lat/0206012

23. D. Thompson et al., Phys. Rev. Lett. 79 (1997) 1630; S. U. Chung et al., Phys. Rev. D60 (1999) 092001; D. Adams et al., Phys. Rev. Lett. 81 (1998) 5760; E.I.Ivanov etal., Phys. Rev. Lett. 86 (2001) 3977

24. C. Michael, Proc. Heavy Flavours 8, Southampton, (ed. P. Dauncey and C. Sachrajda), JHEP, PRHEP-hf8/001, 1-10 (2000); hep-ph/9911219.

25. UKQCD Collaboration, C. McNeile, C. Michael and P. Pennanen, Phys. Rev. D65, (2002) 094505 